\title{
Transplantation of donor grafts with defined ratio of conventional and regulatory $T$ cells in HLA-matched recipients
}

Everett H. Meyer, ${ }^{1,2}$ Ginna Laport, ${ }^{3}$ Bryan J. Xie, ${ }^{1}$ Kate MacDonald, ${ }^{1}$ Kartoosh Heydari, ${ }^{2}$ Bita Sahaf, ${ }^{1}$ Sai-Wen Tang, ${ }^{1}$ Jeanette Baker, ${ }^{1}$ Randall Armstrong, ${ }^{1}$ Keri Tate, ${ }^{4}$ Cynthia Tadisco, ${ }^{4}$ Sally Arai, ${ }^{1}$ Laura Johnston, ${ }^{1}$ Robert Lowsky, ${ }^{1}$ Lori Muffly, ${ }^{1}$ Andrew R. Rezvani, ${ }^{1}$ Judith Shizuru, ${ }^{1}$ Wen-Kai Weng, ${ }^{1}$ Kevin Sheehan, ${ }^{1}$ David Miklos, ${ }^{1}$ and Robert S. Negrin ${ }^{1}$

'Division of Blood and Marrow Transplantation, Stanford University, Stanford, California, USA. ${ }^{2}$ Cell Therapy Facility, Stanford Health Care, Stanford, California, USA. ${ }^{3}$ Tempest Therapeutics, San Francisco, California, USA. ${ }^{4}$ Laboratory for Cell and Gene Medicine, Stanford University, Palo Alto, California, USA.

BACKGROUND. In preclinical murine and early clinical studies of hematopoietic cell transplantation, engineering of donor grafts with defined ratios of $\mathrm{CD} 4^{+} \mathrm{CD} 25^{+} \mathrm{FoxP3}{ }^{+}$Tregs to conventional T cells (Tcons) results in the prevention of graft-versus-host disease and improved immune reconstitution. The use of highly purified primary graft Tregs for direct cell infusion has potential advantages over impure immunomagnetic selection or culture expansion, but has not been tested clinically. We performed a phase I study of the timed addition of CD34-selected hematopoietic stem cells and Tregs, followed by Tcons for the treatment of patients with high-risk hematological malignancies.

METHODS. We present interim evaluation of a single-center open phase I/II study of administration of human leukocyte-matched Tregs and CD34-selected hematopoietic cells, followed by infusion of an equal ratio of Tcons in adult patients undergoing myeloablative hematopoietic stem cell transplantation (HCT) for high-risk or active hematological malignancies. Tregs were purified by immunomagnetic selection and high-speed cell sorting.

RESULTS. Here we report results for the first 12 patients who received Tregs of between $91 \%$ and $96 \%$ purity. Greater than grade II GVHD was noted in 2 patients in the first cohort of 5 patients, who received cryopreserved Tregs, but neither acute nor chronic CVHD was noted in the second cohort of 7 patients, who received fresh Tregs and single-agent CVHD prophylaxis. Patients in the second cohort appeared to have normal immune reconstitution compared with patients who underwent transplantation and did not develop GVHD.

CONCLUSION. Our study shows that the use of highly purified fresh Tregs is clinically feasible and supports continued investigation of the strategy.

TRIAL REGISTRATION. ClinicalTrials.gov NCT01660607.

FUNDING. NIH NHBLI R01 HL114591 and K08HL119590.

Mas a sponsored research

agreement with Orca Biosystems

Inc. initiated in 2018. EHM and RSN

have declared methodology for Treg

selection to Stanford University, which

submitted a patent application.

Copyright: $\Subset 2019$ American Society for Clinical Investigation

Submitted: January 7, 2019

Accepted: April 2, 2019

Published: May 2, 2019.

Reference information: /CI Insight.

2019;4(9):e127244. https://doi.

org/10.1172/jci.insight.127244.

\section{Introduction}

Immunosuppressive or cytotoxic medications continue to be the main method to control alloreactivity following hematopoietic stem cell transplantation (HCT). One area of active study is the development of nontoxic strategies that exploit natural immunoregulatory pathways to facilitate posttransplantation immune reconstitution without causing graft-versus-host disease (GVHD) (1-3). This includes donor graft engineering to select or enrich for immunoregulatory populations.

Tregs are a $\mathrm{T}$ cell subset that can regulate immune responses. They are defined by high surface expression of CD4 and CD25, low-level expression of CD127 (IL-7R), and intracellular expression of the canonical 
transcription factor FoxP3 $(4,5)$. Several murine models and initial clinical studies have suggested that enriching donor grafts with donor Tregs could improve HCT by facilitating immune reconstitution and reducing the risk of GVHD while retaining graft-versus-tumor (GVT) effects $(2,6-8)$.

Different strategies to enrich for Tregs in donor grafts have been tested in patients. One study in haploidentical transplantation used donor Treg enrichment by $\mathrm{CD} 25^{+}$microbead selection, with results suggesting the approach both was safe and appeared to facilitate GVT (6). Enrichment of Tregs with this strategy has variable cell yields, and the product was partially purified, which could potentially limit the options for additional modification of Tregs to enhance efficacy, such as transient or permanent genetic modification. To investigate the safety and feasibility of highly purified Treg graft engineering, we developed a tandem selection strategy using immunomagnetic selection and high-speed flow cytometric sorting to select $\mathrm{CD} 4^{+} \mathrm{CD} 25^{+} \mathrm{CD} 127^{\text {lo }}$ Tregs for infusion.

A major question in the field of Treg therapy is to determine the sufficient dose of Tregs needed, especially in relation to the treatment of different disease states. In the case of HCT, since patients receive intensive chemotherapy or radiotherapy that is both myelo- and lymphodepleting, it has been suggested that relatively few Tregs could have a large effect as compared with other clinical contexts. In murine models, mice generally receive a 1:1 ratio of Tregs to conventional T cells (Tcons), but the dose equivalent per kg of Tregs is much greater than is feasible through purification and enrichment of graft Tregs alone. Further, in the lymphodepleted environment, Tregs expand rapidly (9), and by infusing these cells prior to Tcons, a strategy that can be readily employed in the setting of allogeneic HCT relative in vivo expansion of Tregs can be accomplished. In murine models, this strategy resulted in improved immune reconstitution (9).

In trials using defined ratios of Tregs to Tcons, recipients receive a smaller number of $\mathrm{T}$ cells and accompanying graft cells than in normal standard-of-care grafts. Trials evaluating whether doses obtainable by purification alone provide evidence of clinical safety, sufficient immune reconstitution, or efficacy help to clarify whether alternative strategies may be required in HCT or other clinical contexts. Alternative strategies include ex vivo cell expansion; in vivo cell expansion through, for example, IL-2 supplementation; or genetic modification of Tregs. Early reports on the use of third-party in vitro expanded cord blood Tregs also suggested this approach was safe in HCT; however, there is significant cost associated with cell expansion, and third-party cells may not persist in recipients $(1,10,11)$.

We report interim results of our phase I dose escalation and early phase II dose extension study using this graft engineering approach for patients with high-risk or active hematologic malignancies undergoing allogeneic myeloablative HCT from a human leukocyte antigen-matched (HLA-matched) donor (Figure 1). While this is seemingly a simple question in Treg therapy, during the implementation of this phase I/II trial and in part due to early results leading to further preclinical studies by our group and others $(6,7)$, our results suggest that cryopreservation and thaw prior to infusion may not be as successful a strategy as the use of fresh Tregs in the HCT setting. The present results suggest that donor graft engineering with defined ratios of fresh Tregs to conventional $\mathrm{CD}^{+} \mathrm{T}$ cells is both safe and feasible.

\section{Results}

Patient demographics. Table 1 shows the primary disease and disease status of patients at the time of HCT. All recipients had high-risk or active disease, including acute myeloid leukemia $(n=5)$, acute lymphoblastic leukemia $(n=2)$, refractory non-Hodgkin lymphoma $(n=2)$, myeloproliferative disease $(n=1)$, chronic myeloid leukemia $(n=1)$, and myelodysplastic syndrome (MDS) $(n=1)$.

Dose escalation. Figure 1 shows the conditioning and GVHD prophylaxis schema, before and after protocol modification (occurring after the fifth patient treated; further protocol schematic in Supplemental Figure 1; supplemental material available online with this article; https://doi.org/10.1172/jci.insight.127244DS1). The Treg dose escalation was established as the ratio of Tregs to Tcons, with the initial cohort target dose of $1.0 \times$ $10^{6}$ Tregs $/ \mathrm{kg}$ and $3.0 \times 10^{6} \mathrm{CD}^{+}$Tcons $/ \mathrm{kg}$ in the first cohort of 3 patients. Since GVHD was observed in one patient, according to the predefined dose-escalation plan (Supplemental Figure 2), the group underwent expansion to an additional 3 subjects at a Treg/Tcon ratio of $1: 1$ of $1 \times 10^{6}$ Tregs $/ \mathrm{kg}$ and $1 \times 10^{6} \mathrm{Tcon} / \mathrm{kg}$. Since no dose-limiting toxicity was observed in these additional subjects, the target dose was escalated to $3.0 \times 10^{6}$ Tregs $/ \mathrm{kg}$ and $3.0 \times 10^{6} \mathrm{CD}^{+}$Tcons $/ \mathrm{kg}$. After no dose-limiting toxicity was observed in 3 additional patients, the maximally tolerated target dose was established, which was also the maximal technical dose of Tregs that could be obtained from 2 apheresis collections, and an additional 3 patients were accrued at this dose level. In patients in whom achieving $3 \times 10^{6} \mathrm{Treg} / \mathrm{kg}$ was not feasible, the cell dose achieved was administered. 


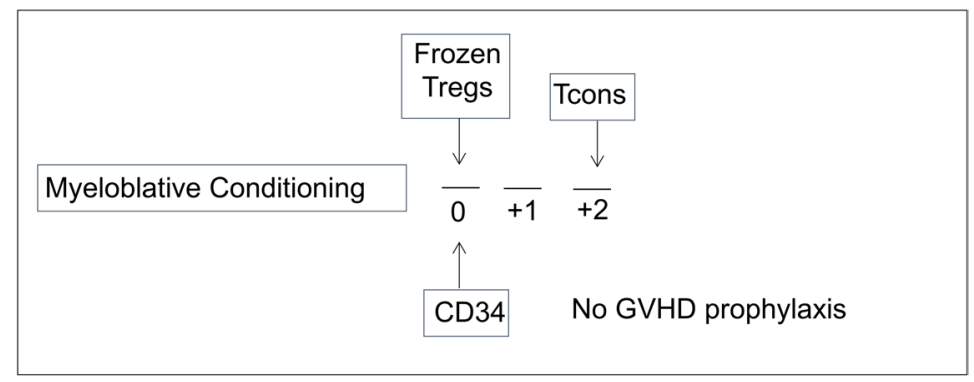

\section{Modified Protocol}

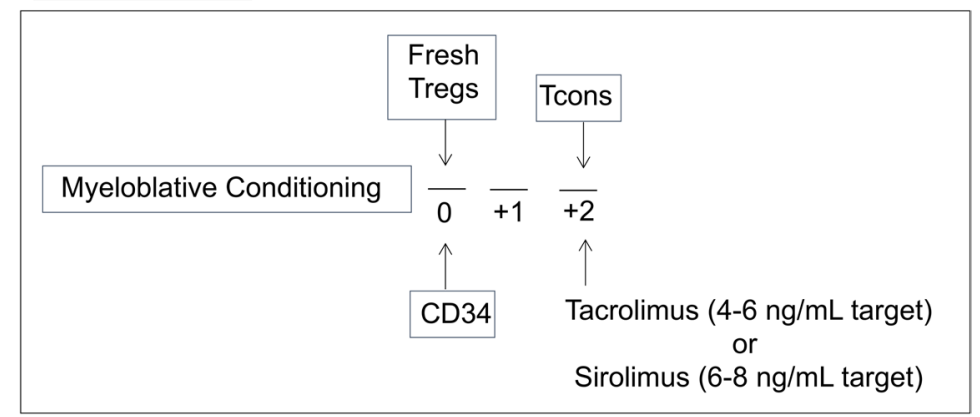

Figure 1. Protocol schema. The initial protocol utilized frozen Tregs and no GVHD prophylaxis. After the accrual of the first 5 patients between September 2011 and May 2015 (initial protocol), the protocol underwent a review and revision in November 2015 (modified protocol) based on preclinical data that frozen and thawed Tregs do not function as well as freshly isolated cells.

Donor graft and Treg properties. Tregs were purified from G-CSF-mobilized products by immunomagnetic selection $\left(\mathrm{CD} 25^{+}\right)$on the CliniMACS Plus System (Miltenyi Biotec), followed by flow cytometric cell sorting on an Influx high-speed cell sorter (BD Biosciences) by gated selection of the CD4 $4^{+} \mathrm{CD} 127^{\text {lo }}$ population. For a representative analysis of cells collected after $\mathrm{CD} 34^{+}$selection, $\mathrm{CD} 25^{+}$cell selection, and $\mathrm{CD} 4^{+} \mathrm{CD} 127^{+}$cell sorting analyzed for $\mathrm{CD} 4$ and FoxP3 expression, see Figure 2A. As shown, high-purity Tregs were obtained. These cells were highly suppressive in the mixed lymphocyte reaction (Figure 2B and Supplemental Figure 3). All subjects at the $1.0 \times 10^{6} / \mathrm{kg}$ Treg dose received this target dose of Tregs; however, not all subjects received a target dose of $3.0 \times 10^{6} \mathrm{Tregs} / \mathrm{kg}$. Table 2 shows the cell dose yields of subjects, with a median of $2.5 \times 10^{6} \mathrm{Tregs} / \mathrm{kg}$ administered (range $2.4 \times 10^{6}$ to $3.0 \times 10^{6}$ Tregs/ $\mathrm{kg}$ ). Median Treg recovery was $67 \%$ (range 50.7\%-87.8\%), and median Treg purity was $94 \%$ (91\%-96\%), based on $\mathrm{FoxP}^{+}$expression by intracellular cytokine staining. All but one patient achieved a CD $34^{+}$dose greater than $2.0 \times 10^{6}$ cells $/ \mathrm{kg}$ with a median dose of $3.9 \times 10^{6} 10^{6} \mathrm{cells} / \mathrm{kg}\left(\right.$ range $1.2 \times 10^{6}$ to $15.9 \times 10^{6}$ ).

Treg infusion, engraftment, and relapse. Treg infusions were completed on day 0 for all patients. No infusion reactions were observed. All 12 of the subjects achieved primary full donor engraftment; however one subject receiving the lowest dose level $\left(1.0 \times 10^{6}\right.$ Tregs $/ \mathrm{kg}$ and $\left.1.0 \times 10^{6} \mathrm{CD}^{+} \mathrm{Tcons} / \mathrm{kg}\right)$ with high-risk RAEB-2 MDS had secondary graft failure. Including this subject, neutrophils reached $1.0 \times 10^{9} / 1$ at a median of 11 days (10-16). Platelets reached $50 \times 10^{9} / 1$ at a median of 16 days (9-25; Supplemental Figure 4). Although there was a limited number of patients, there appeared to be no differences between dose levels. Prior to protocol modification, three of five patients treated achieved a complete remission ( 2 of whom remain in remission more than 1 year at the time of writing of the manuscript). Following protocol modification, 5 of 7 patients achieved a complete remission (4 of whom remained in remission more than 1 year at the time of writing), and no acute or chronic GVHD was observed.

Posttransplant complications, GVHD, and infections. Patient outcomes are shown in Table 3. In the initial patient at a Treg/Tcon ratio of 1:3, acute GVHD grade III was observed. Therefore, the ratio was readjusted according to the protocol to $1: 1$ at a dose of $1 \times 10^{6} / \mathrm{kg}$ for both Tregs and Tcons. Of the first 3 subjects treated at this dose level, 1 case of acute GVHD was observed (grade III). An additional 2 patients were treated at this ratio. One additional patient developed acute grade I skin GVHD. All cases of GVHD were 


\section{Table 1. Patient characteristics}

\begin{tabular}{|c|c|c|c|c|c|c|c|}
\hline Subject & Age & Sex & CMV (D/R) & Cohort & Conditioning & GVHD PPx & Disease \\
\hline \multicolumn{8}{|c|}{ Initial protocol: frozen Tregs } \\
\hline 5126 & 56 & M & $-1+$ & 1 & TBI/Су/VP-16 & None & AML \\
\hline 5534 & 65 & M & $-1-$ & $1 \mathrm{~A}$ & TBI/Cy/VP-16 & None & MDS, RAEB2 \\
\hline 5902 & 45 & $\mathrm{~F}$ & $+/+$ & $1 \mathrm{~A}$ & BCNU/VP-16/Cy & None & $\begin{array}{c}\text { Gamma delta } \\
\text { NHL }\end{array}$ \\
\hline 6666 & 49 & M & $-/-$ & $1 \mathrm{~A}$ & BCNU/VP-16/Cy & Sirolimus & DLBCL NHL \\
\hline 6708 & 42 & M & $+1-$ & $2 \mathrm{~A}$ & $\mathrm{Bu} / \mathrm{Cy}$ & Sirolimus & $\mathrm{FLT3}+\mathrm{AML}$ \\
\hline 6784 & 34 & M & $+/+$ & $2 \mathrm{~A}$ & TBI/Сy/VP-16 & Sirolimus & ETP-ALL \\
\hline 6820 & 20 & M & $+/+$ & $2 \mathrm{~A}$ & $\mathrm{Bu} / \mathrm{Cy}$ & Tacrolimus & ALL \\
\hline 6857 & 54 & M & $+/-$ & $2 \mathrm{~A}$ & $\mathrm{Bu} / \mathrm{Cy}$ & Tacrolimus & CML blast crisis \\
\hline
\end{tabular}

PPx, prophylaxis; TBI, total body irradiation; Cy, cytoxan; VP-16, etoposide; Bu, busulfan; AML, acute myeloid leukemia; CR, complete remission; MDS, myelodysplastic syndrome; RAEB2, refractory anemia of excess blasts 2; NHL, non-Hodgkin lymphoma; ETP-ALL, early T cell precursor acute lymphoplastic leukemia; MRD, minimal residual disease; CML, chronic myeloid leukemia; MF, myelofibrosis.

steroid responsive. Preclinical murine modeling demonstrated that Tregs that undergo cryopreservation have some loss of in vivo function due to shedding of CD62L, which is critical for lymph node homing (10). Therefore, the protocol was modified to use only fresh Tregs and Tcons and to include low-dose single-agent GVHD prophylaxis with sirolimus, since this agent has been shown to be protective of Tregs. In those clinical situations where the risk of sinusoidal obstruction syndrome (SOS) could be increased with sirolimus, low-dose tacrolimus was substituted. Following protocol modification, no additional cases of GVHD were observed in the subsequent 7 patients, although one had transaminitis and was treated for possible hepatic GVHD with a short course of steroids. However, the transaminitis was determined most likely related to a number of possible medications that were changed at the same time, with quick resolution of the transaminitis and no elevation of total bilirubin.

A total of 11 patients achieved survival greater than 6 months and could be evaluated for chronic GVHD. At a median follow-up of 481 days (range 212-1887 days), 2 of the 11 subjects, both of whom were in the initial protocol cohort, developed chronic GVHD. Both had prior grade III acute GVHD, and chronic GVHD manifestations were limited to skin and responsive to steroids. After protocol modification, no patients in the second cohort had developed chronic GVHD at the time of writing of the manuscript.

After protocol modification, 1 patient who underwent busulfan and cyclophosphamide conditioning and received sirolimus for GVHD prophylaxis had mild SOS and was treated to resolution with defibrotide and supportive care. Eleven of 12 patients experienced mucositis (4 with grade I, 3 with grade II, and 4 with grade III).

Posttransplantation immune reconstitution. In order to evaluate immune reconstitution in patients enrolled in this study, we identified a comparison standard-of-care control cohort of 5 concurrent patients who received myeloablative HCT and who had no acute or chronic GVHD or relapse. Using flow cytometry, we quantified immune cell subsets from frozen and thawed PBMCs at standardized time points after HCT (Figure 3). Patients in the initial cohort showed relatively poor T cell, B cell, NK cell, and Treg reconstitution compared with patients treated under the modified protocol or standard of care. This evaluation of immune reconstitution was influenced by both the small patient numbers and the fact that most patients in the initial cohort received systemic steroids for proven or suspected acute and chronic GVHD.

Patients treated on the modified protocol with fresh Tregs appeared to have generally comparable immune reconstitution in T cell, B cell, NK cell, DC, and monocyte populations as compared with standard-of-care controls without GVHD (Figure 3). In contrast, the absolute number of iNKT cells was significantly lower in the first 6 months in both the initial- and modified-protocol patient cohorts when compared with controls (Figure 3; 2-tailed Student's $t$ test, $P<0.01$ ). 

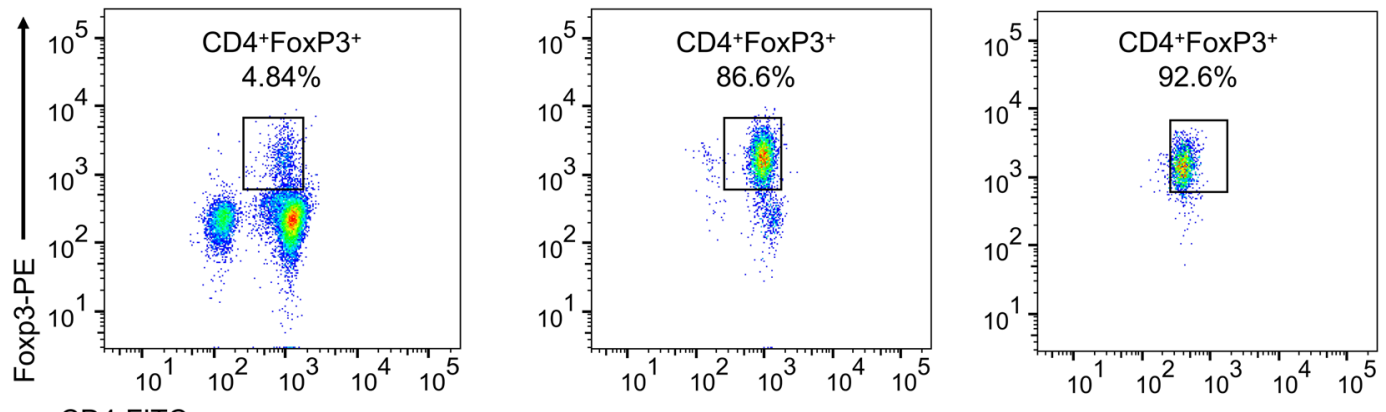

CD4-FITC

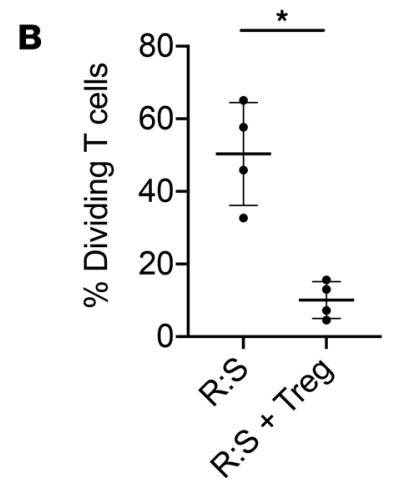

Figure 2. Cell selection and purification of Tregs. (A) Enrichment of Tregs from peripheral blood G-CSF-mobilized progenitor cells, following depletion of CD34+ cells by immunomagnetic selection (left), selection of CD25+ by immunomagnetic selection (middle), and purification by FACS sorting of CD4+CD127/0 $C 25^{+}$cells (right panel). Representative plots from 1 patient expressed as CD4+ and intracellularly stained FoxP3. (B) Suppression of the MLR by purified Tregs. The percentage of CFSE ${ }^{\text {dim }}$ populations of T cell-cultured HLA-mismatched PBMCs with/without Tregs (1:1 ratio) in alloMLR, analyzed by FACS. Results are mean \pm SD from 4 patients evaluated; $P<0.01$, 2-tailed Student's $t$ test.

Posttransplantation Treg reconstitution. Using flow cytometry, immune reconstitution in terms of percentage and absolute value of Tregs following HCT appeared to be comparable in patients treated with fresh Tregs on the modified protocol and in standard-of-care controls who had not developed acute or chronic GVHD (Figure 3; 2-tailed Student's $t$ test, $P=0.01$ ). Phenotypic characterization of Tregs by flow cytometry showed no statistical differences in the expression of the surface receptors CTLA-4 (CD152), PD-1, CD62L, CD45RO, and FoxP3 (Figure 4 and Supplemental Figure 5). This was confirmed by mass cytometry (CyTOF), with the additional observation that there appeared to be no increases in intracellular IFN- $\gamma$ or TNF- $\alpha$ expression ex vivo and without stimulation.

In order to assess TCR repertoire reconstitution of the Treg compartment following HCT, we used FACS to purify $\mathrm{CD} 4{ }^{+} \mathrm{CD} 25^{+} \mathrm{CD} 127^{\text {lo }}$ Tregs from patients on approximately day 90 after transplant. Between 2700 and 48,000 Tregs were isolated. Using RNA as a template, T cell receptor repertoire sequencing (TCR Rep-Seq) was performed. There were no statistical differences in the diversity of Tregs following either the standard-of-care or the modified clinical trial protocol (Figure 4C).

\section{Discussion}

Preclinical murine studies have demonstrated that engineering the donor graft with defined numbers of Tregs and Tcons may improve HCT outcomes by preventing GVHD and enhancing immune reconstitution $(2,3,11)$. In these murine models, GVT effects were maintained possibly through Treg control of alloreactive $\mathrm{T}$ cell proliferation, without interfering with Tcon activation and function (2). We aimed to translate these observations into the clinic.

There are several features of our study notably differing from other studies exploring the biological role of Tregs in allogeneic HCT. First, we utilized highly purified populations of Tregs isolated by 
Table 2. Treg selection characteristics: patient dosing, Treg purity and sort recovery

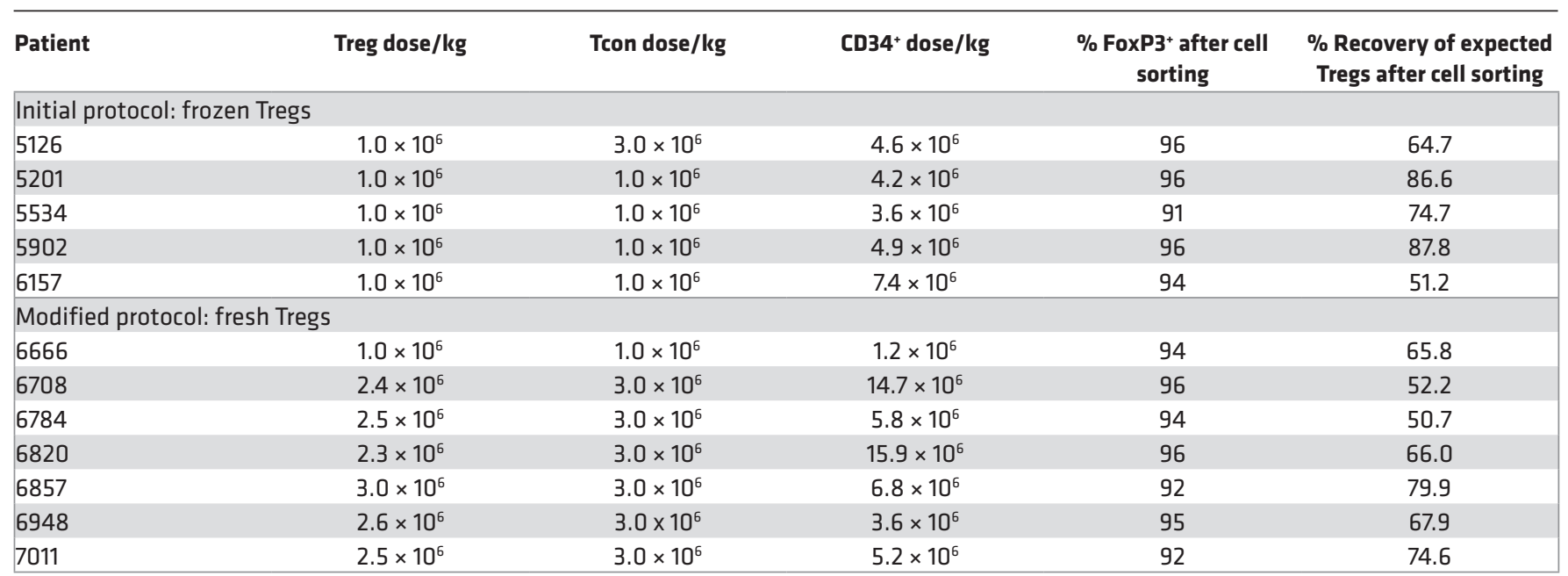

immunomagnetic cell isolation and high-speed cell sorting. Tregs are rare cells, constituting approximately $1 \%-4 \%$ of peripheral blood lymphocytes. We obtained highly purified Tregs in excess of $91 \%$ FoxP3 expression in all cases, with the upper limit of Treg dose that can be reliably obtained after 2-day collection being between $2.5 \times 10^{6}$ and $3 \times 10^{6} \mathrm{Tregs} / \mathrm{kg}$. Our early results would suggest that this dose of Tregs is sufficient in the setting of myeloablative conditioning to have an effect in immune reconstitution.

The majority of studies in the literature using Tregs in the clinical realm are in the haploidentical or cord blood transplant setting. Our study is the first to our knowledge to use HLA-matched donors, which are the most commonly utilized donor recipient pairs in the HCT setting. In this study, only HLA-matched sibling donors were included. However, moving forward, patients with an available sibling or unrelated donor will be eligible.

A third differentiating feature was the clinical data obtained regarding the use of fresh as compared with cryopreserved cells. During the course of this clinical study, investigation of murine and human Tregs at our center and others clarified that cryopreservation and subsequent thawing of Tregs resulted in cleavage of CD62L, which is critical for in vivo Treg function (12). The experience of our first 5 patients, who received cells thawed at bedside and showed manifestations of GVHD and overall signs of impaired immune reconstitution, is consistent with reduced functionality of Tregs. These results necessitated a change in our clinical manufacturing to the production and infusion of fresh Tregs.

A major finding of our study is that the use of fresh Tregs and low-dose single-agent immunosuppression resulted in no acute or chronic GVHD following myeloablative HCT, in contrast to our initial trial participants, who received cryopreserved Tregs. However, due to the small number of patients treated, it is not possible to make definitive conclusions. Sirolimus is the preferred low-dose single-agent immunosuppressive therapeutic, as this drug has been shown to be permissive of Treg function in vitro (12). However, we had 1 case of SOS in a patient conditioned with busulfan and cyclophosphamide. Following this SOS event, only patients conditioned with total body irradiation (TBI) were eligible to receive sirolimus; otherwise low-dose tacrolimus was substituted. While tacrolimus is known to adversely affect Treg function, the early results of this trial suggest that it can be used effectively in combination, at least in the HCT setting. The combination of tacrolimus with methotrexate is one of the most commonly administered GVHD prophylaxis regimens. The removal of methotrexate GVHD prophylaxis can be beneficial, with the potential for less mucositis, earlier neutrophil engraftment, and less risk of organ damage or long-term risk of secondary malignancy. It remains to be tested whether inclusion of immunosuppressive drugs is necessary when utilizing fresh Tregs.

We observed no obvious impairment in graft-versus-leukemia/tumor effects in trial patients with a high degree of disease burden, all of whom had high-risk and often refractory hematological malignancies, although the numbers included in this trial were small. A trend toward enhanced GVT effects has been 
Table 3. Patient outcomes

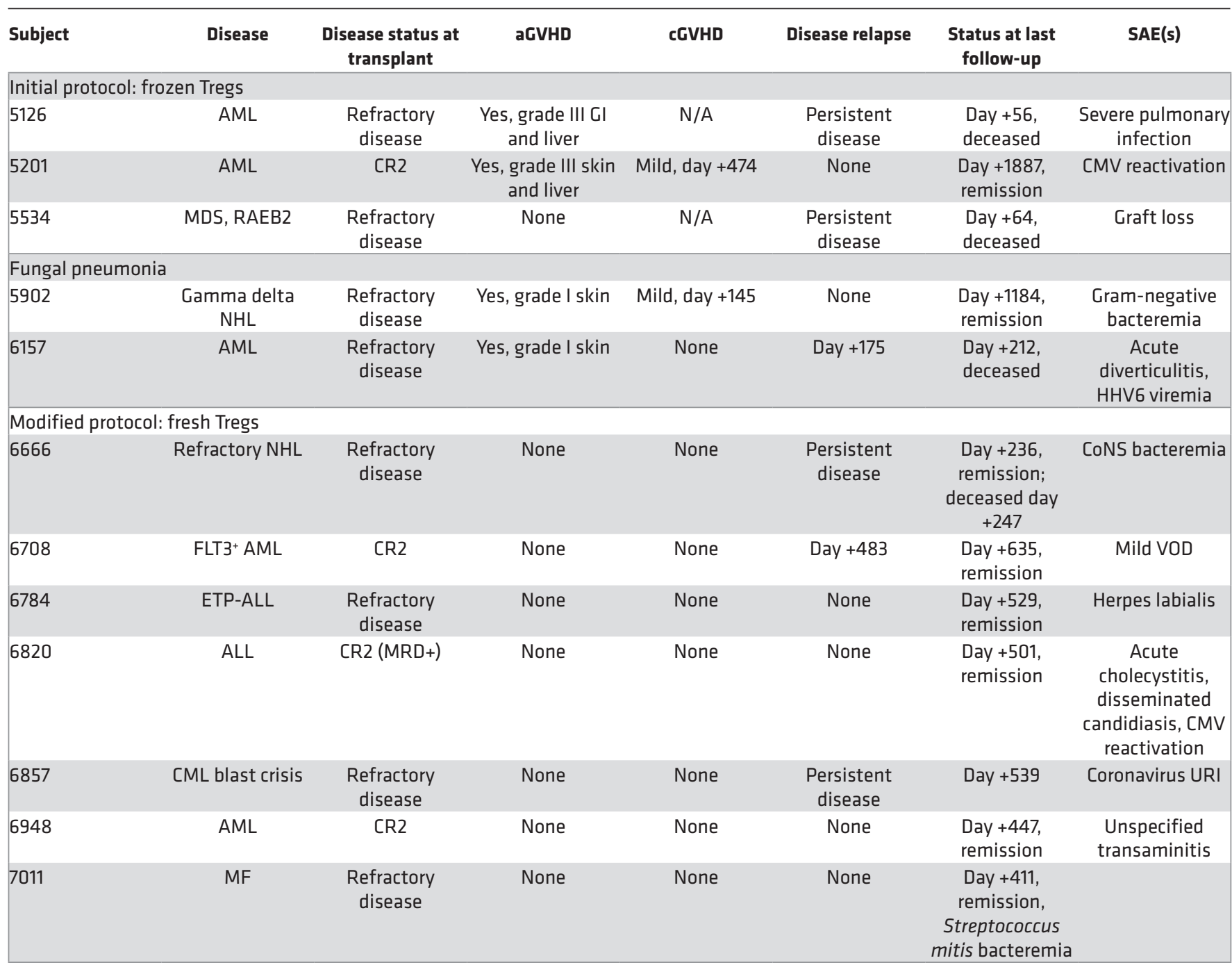

aGVHD, acute GVHD; CGVHD, chronic GVHD; GI, gastrointestinal; ML, acute myeloid leukemia; CR, complete remission; MDS, myelodysplastic syndrome; RAEB2, refractory anemia of excess blasts 2; NHL, non-Hodgkin lymphoma; CoNS, coagulase-negative staphylococci; VOD, veno-occlusive disease; ETPALL, early T cell precursor acute lymphoplastic leukemia; MRD, minimal residual disease; CML, chronic myeloid leukemia; MF, myelofibrosis; URI, upper respiratory infection.

suggested in other clinical studies of Tregs and Tcons (13). We saw few viral infections, such as CMV reactivation or end organ involvement, in our treated subjects, and EBV reactivation was not observed.

Four of 5 patients treated on the initial protocol demonstrated acute GVHD, with 2 patients demonstrating clear histologic and clinical diagnosis of grade III disease, further suggesting that the cryopreserved Tregs have compromised function. Two additional patients had grade I skin GVHD with nonspecific histology, and 1 additional patient was noted to have transient rashes not attributed to GVHD. All of these cases were early in their HCT course, and patients with grade I GVHD and rash could be considered similar in nature to descriptions of early posttransplant inflammation or engraftment syndrome or GVHD. Notably, we saw no classically confirmed acute or chronic GVHD in the 7 patients enrolled on the modified protocol with fresh Tregs. The historical rates of $37.2 \%$ acute and 58.0\% chronic GVHD were observed in our concurrent standard-of-care population with matched related donors $(n=168)$.

Immune reconstitution appeared robust in those patients treated on the modified protocol. $\mathrm{CD} 4^{+} \mathrm{T}$ cell reconstitution was brisk, with patients recovering more than $200 \mathrm{CD} 4^{+} \mathrm{T}$ cells $/ \mu \mathrm{l}$ by 60 days after transplant (Figure 3). Treg phenotypic and TCR repertoire reconstitution was comparable to a cohort of concurrent 

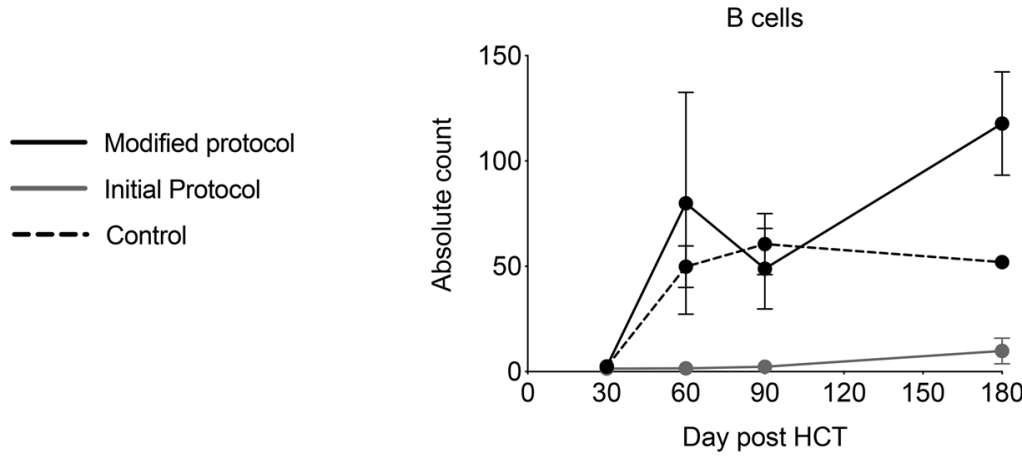

CD4+ cells
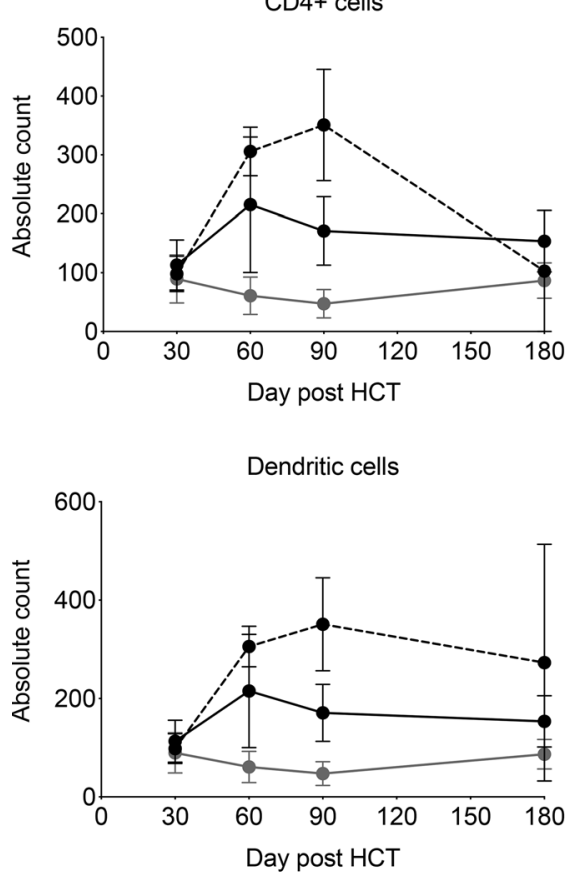
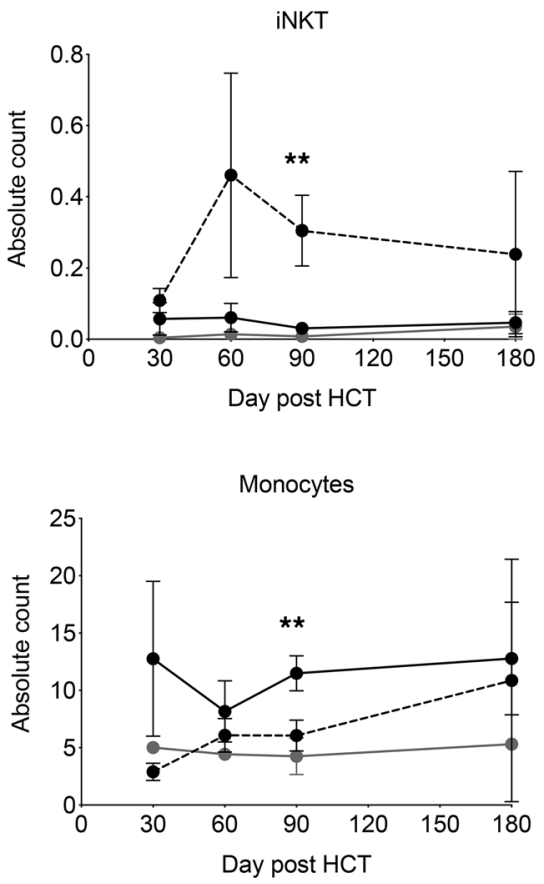

T cells

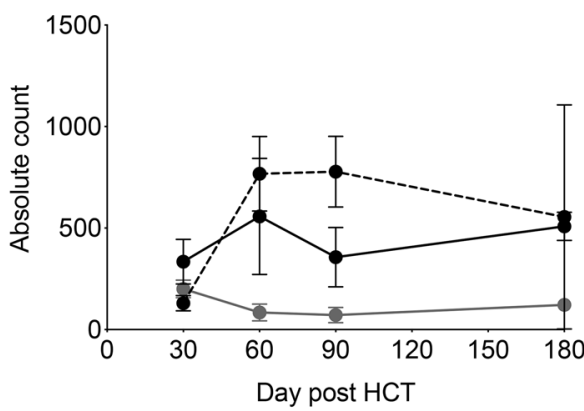

Treg

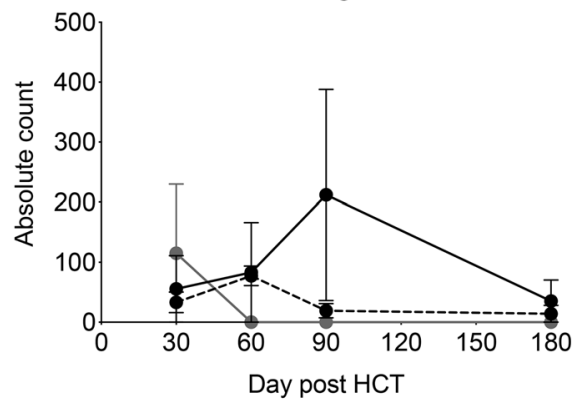

NK cells

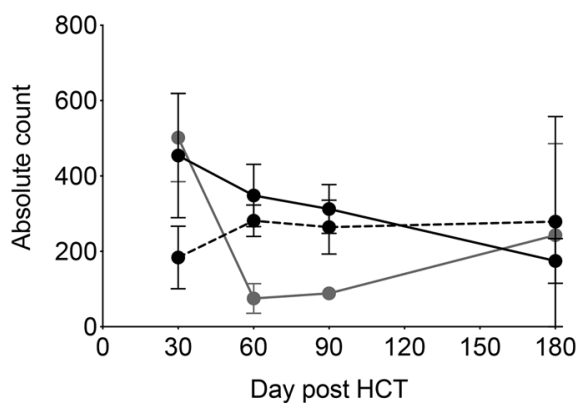

Figure 3. Reconstitution of immune cell populations following hematopoietic cell transplantation. Patients on the modified protocol demonstrated improved absolute cell counts in B cells, T cells, and Tregs compared with initial patients 180 days after hematopoietic cell transplantation (HCT). Counts are shown in cells $/ \mathrm{ml}$ of blood. ${ }^{*} P<0.05$, Mann-Whitney $U$ test, between control patients and patients on the modified protocol. Error bars represent mean \pm SEM. $n=7$ for modified protocol; $n=5$ for control; and $n=3$ for initial protocol.

standard-of-care patients who did not have complications (Figure 4). Our results suggest that there is effective Treg reconstitution with graft engineering. In contrast, patients who received cryopreserved Tregs had impaired immune and Treg reconstitution, but this could be confounded by age, low initial cell doses, steroid dosing, and other factors.

The lack of invariant NKT (iNKT) cell reconstitution in patients receiving engineered Treg grafts suggests that reconstitution of this immune cell subset in the first 100 days after HCT may depend upon iNKT cells being present in the donor graft. Given the potential importance of iNKT cells as positively correlated to better HCT outcomes (15) and interaction with Tregs, future efforts at graft engineering might include this subset specifically, especially as they may act synergistically with Tregs (15).

Our studies might indicate that ex vivo expanded Tregs may not be needed in the HCT setting, or if they are used, they could be used effectively in relatively small numbers. Likewise, the present graft engineering study lays the foundation to develop strategies to genetically modify Tregs or other graft constituents that could facilitate GVT while preventing GVHD and infectious complications (8, 16-19). HCT is used in other contexts outside of cancer therapy, including combined organ and HCT transplantation to induce immune tolerance to organ grafts. One potential application is the incorporation of Tregs into HCT to augment donor chimerism and tolerance $(20,21)$. The feasibility of this approach is steadily increasing with accumulating evidence, such as provided in this study, demonstrating the safety of Treg graft engineering. 

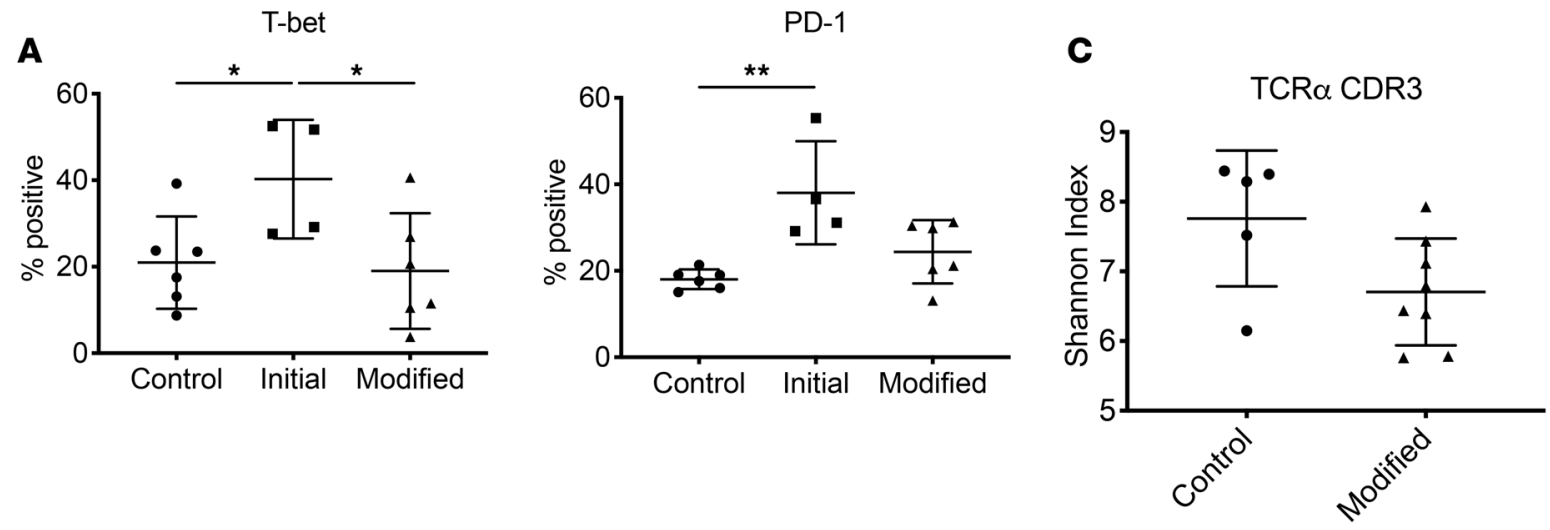

Ki-67
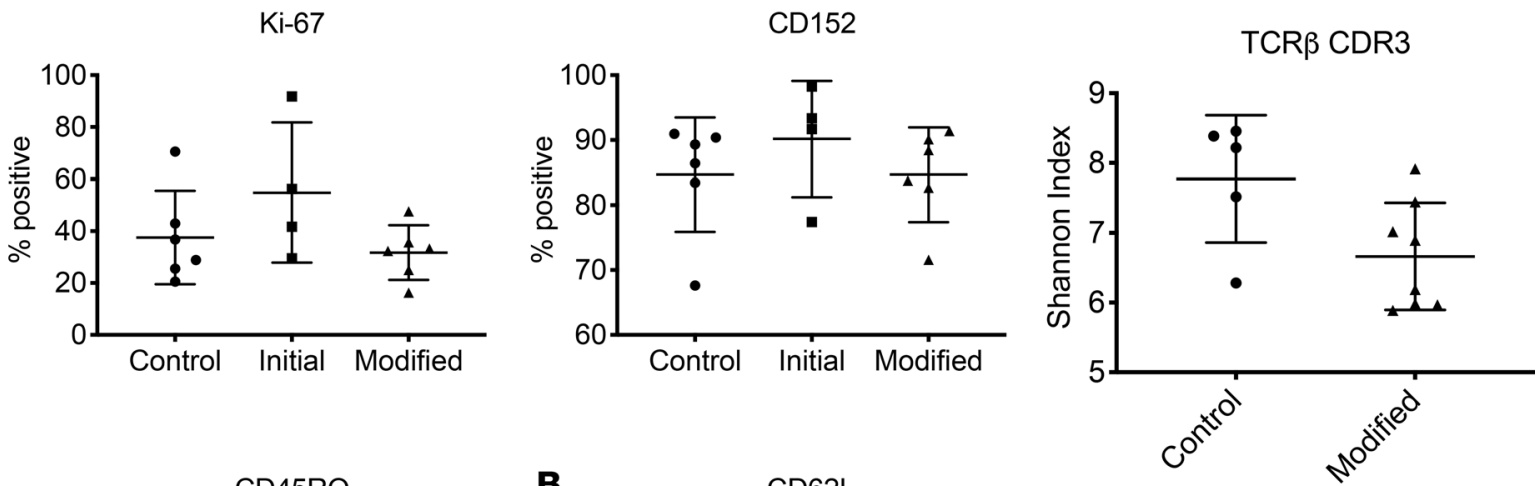

CD45RO

B

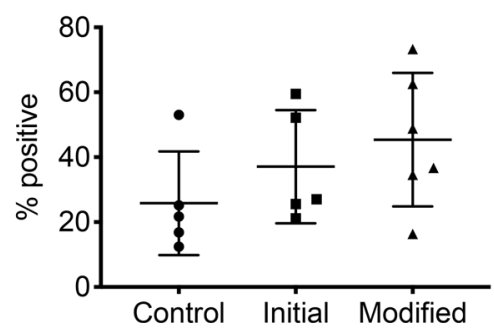

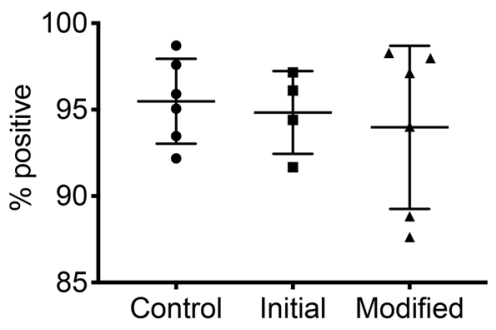

Figure 4. Immune reconstitution and TCR diversity of peripheral blood Tregs. Reconstitution of naive Tregs as quantified by (A) CyTOF and (B) flow cytometric staining, both gated on $\mathrm{CD} 4^{+} \mathrm{CD} 25^{+} \mathrm{CD} 127^{\circ}$. Patients on the modified protocol $(n=6)$ are compared with standard-of-care controls $(n=5)$ and initial protocol $(n=5)$ participants at 60 days after HCT. (C) TCR $\alpha$ and $-\beta$ CDR3 repertoire diversity of FACS-purified CD4+CD25+CD12710 Tregs at day 90 after HCT for patients on the modified protocol $(n=7)$ versus standard-of-care controls $(n=5)$. ${ }^{*} P<0.05$ and ${ }^{* *} P<0.01$ respectively, Mann-Whitney $U$ test, between control patients and patients on the modified protocol. Error bars represent mean \pm SEM.

In summary, this study demonstrates that high-purity enrichment of Tregs can be accomplished on a clinical scale and that fresh Tregs are preferable in the HCT setting. Preliminary evidence shows that this approach is feasible and results in low rates of acute and chronic GVHD using HLA-matched donor-recipient pairs. Further, the doses of Tregs and Tcons have been defined for subsequent clinical studies of larger numbers of patients.

\section{Methods}

Study design. The primary objective of the study was to determine the efficacy, safety, and feasibility of administration of Tcons and Tregs using a T cell-depleted graft; the maximum tolerated dose of infused Tregs and Tcons; and 1-year event-free survival (EFS) after HCT. Safety was assessed as any grade II or greater serious adverse event (SAE) attributed to treatment, and specific dose-limiting toxicity was defined as acute GVHD greater than grade II; grade IV neutropenia lasting to 28 days after HCT; and grade III-V cytokine/release syndrome/acute infusion reactions.

Inclusion criteria for enrollment were as follows: acute leukemia (primary refractory, CR1 with minimal residual disease-positive [MRD-positive] or high-risk features, beyond CR1), chronic myelogenous leukemia (accelerated or blast phase), MDSs (Int-2 or high-risk), myeloproliferative disorders, 
non-Hodgkin lymphoma with poor risk features not suitable for autologous HCT, age $\leq 60$ years, availability of an HLA-matched related donor, and no prior myeloablative therapy or HCT.

The original clinical protocol schema and modified schema are shown in Figure 1. In alignment with previous preclinical and clinical studies, Tregs were administered with the donor graft 2 days prior to Tcons $(2,6)$. After accrual of the first cohort of 5 patients on the original protocol, GVHD was noted, and preclinical studies demonstrated that cryopreservation of Tregs adversely affects function (11). The protocol was amended to utilize fresh rather than frozen Tregs and to introduce targeted-dose single-agent tacrolimus (4-6 $\mathrm{ng} / \mathrm{ml})$ or sirolimus (6-8 $\mathrm{ng} / \mathrm{ml})$ GVHD prophylaxis.

Conditioning regimen and supportive care. The clinical protocol supported the use of different myeloablative conditioning regimens depending on the disease characteristics of enrolled subjects. For acute leukemia, high-risk CML, MDS, and myeloproliferative disorders, conditioning included fractionated TBI (fTBI; 1320 cGy, fractionated over 4 days), VP16 (60 mg/kg, as a single infusion), and cyclophosphamide (60 mg/kg, as a single infusion); or busulfan ( $3.6 \mathrm{mg} / \mathrm{kg}$ q24 initially, infused over 4 days, with targeting to busulfan level of $800-900 \mathrm{nM})$ and cyclophosphamide $(60 \mathrm{mg} / \mathrm{kg}$, per infusion over 2 infusions). For non-Hodgkin lymphoma, conditioning consisted of carmustine $\left(300 \mathrm{mg} / \mathrm{m}^{2}\right)$, VP16 (60 mg/kg), and cyclophosphamide (100 mg/kg).

Subjects on the original protocol received no GVHD prophylaxis; however, after protocol modification subjects could receive either tacrolimus or sirolimus, based on preclinical evidence that sirolimus facilitates Treg function. Following 1 case of SOS in a subject with BU/Cy conditioning, sirolimus GVHD prophylaxis was restricted to subjects with TBI-based conditioning.

Infectious disease prophylaxis consisted of $400 \mathrm{mg}$ acyclovir tid po (for 1 year), single-strength Bactrim (day 30-60), and ciprofloxacin $500 \mathrm{mg}$ bid po (day -2 until engraftment). Viral surveillance via quantitative PCR was performed for EBV (every 2 weeks) and CMV (weekly) starting on day +14 until day +100 .

Graft engineering for CD $34^{+}$HSCs, Tregs, and Tcons. Donor cells were obtained from volunteer donors by apheresis at Stanford Health Care after 5 daily doses of $10 \mathrm{mcg} / \mathrm{kg}$ rhG-CSF (Neupogen, Amgen) using a continuous-flow cell separator (SPECTRA; Cobe BCT). Two consecutive apheresis collections were performed on days 4 and 5 , and the cell products were combined. $\mathrm{CD}_{3} 4^{+}$cells were collected using either the Isolex 300i (Baxter healthcare) or the CliniMACS Cell Selection System (Miltenyi Biotec). The CD34-reduced (flow-through) fractions were retained and used for isolation of donor Tregs. For cell selection, clinical-grade reagents were used under good manufacturing practice conditions within the Blood and Marrow Transplant Cellular Therapy Facility.

$\mathrm{CD} 25^{+}$cells were then selected from the CD34-depleted fraction using bead purification (Miltenyi Biotec). Tcons were obtained from the negative fraction, and the positive fraction was used for Treg purification. $\mathrm{CD} 4^{+} \mathrm{CD} 25^{+} \mathrm{CD} 127^{\text {lo }}$ cells underwent further selection by FACS using a BD Influx cell sorter (BD Biosciences). For clinical sorting, the antibodies were repurified over protein A (or G) columns, held at low $\mathrm{pH}$ for 30 minutes. Antibodies were conjugated with FITC and Alexa Fluor 647, sterile filtered, and stored in single-use aliquots.

Mixed lymphocyte reactions. Enriched $\mathrm{CD}^{+}$and $\mathrm{CD}^{+}$cells $\left(2 \times 10^{5}\right.$, MACS beads; Miltenyi Biotec $)$ from donor peripheral blood or apheresis were set in triplicate in 96-well flat-bottom plates as responders. Responders were labeled with CellTrace CFSE according to the manufacturer's instructions on day 0 (Invitrogen, Molecular Probes) or pulsed with $1 \mu \mathrm{Ci} /$ well $\left(\left[{ }^{3} \mathrm{H}\right]\right.$ thymidine, PerkinElmer) for the last 16 hours of the 5-day assay. Stimulators were a combination of 9 different buffy coats from healthy donors and were irradiated with cesium irradiator $\left({ }^{157} \mathrm{Cs}\right)$ at $33 \mathrm{~Gy}$ and set in responders to stimulators $(\mathrm{R} / \mathrm{S})$ ratios of $1: 0,1: 1,1: 2$, and $1: 4$. Tregs were added as suppressors in responders to suppressors (R/SUP) ratios of 1:1, 1:0.5, 1:0.25, and 1:10. The media (cRPMI) was supplemented with OKT3 (Miltenyi Biotec). Results were read on day 5 by flow cytometry (LSR II, BD Biosciences) or a Beta counter reader (Tomtec, Wallac).

Immunological flow cytometry and CyTOF studies. PBMCs from BMT patients with or without preinfusion of Tregs were recovered from liquid nitrogen. PBMCs were washed with MACS buffer and stained with a pan-PBMC panel or Treg activation panel at $4^{\circ} \mathrm{C}$ for 1 hour. After washing with MACS buffer, the stained cells were fixed with $4 \%$ formaldehyde at $4^{\circ} \mathrm{C}$ overnight. Flow cytometric analysis was performed on an LSR II, and data were analyzed by FlowJo software. Viability staining was performed using $0.5 \mu \mathrm{M}$ cisplatinum (Fluidigm, catalog 201064) for 5 minutes at room temperature. Cell surface antibody master mix was prepared and filtered through $0.2-\mu \mathrm{m}$ filters. Cells were resuspended in the master mix and incubated for 30 minutes. Cells were washed and incubated in the fixing solution prepared in accordance with the 
manufacturer's recommendations (eBioscience, catalog 00-5523). The cells were fixed for 1 hour at room temperature, washed, and then incubated with intracellular antibody mixture in permeabilization buffer (eBioscience, catalog 00-5523) for 1 hour.

Treg TCR receptor sequencing. For TCR repertoire analysis, Tregs were enriched from PBMCs of patients by a 2-step isolation procedure: $\mathrm{CD} 25^{+}$cells were isolated from PBMCs using CD25 MicroBeads II (Miltenyi Biotec) following the manufacturer's instructions. After staining with anti-CD3 (OKT3, BioLegend), -CD4 (OKT4, BioLegend), -CD8 (RPA-T8, BioLegend), -CD25 (BC96, BioLegend), and $-\mathrm{CD} 127$ (A019D5, BioLegend) antibodies, $\mathrm{CD}^{+}, \mathrm{CD}^{+}, \mathrm{CD}^{-}, \mathrm{CD} 25^{+}$, and $\mathrm{CD} 127^{\mathrm{dim}}$ cells were isolated, lysed by TRIzol Plus reagent, and stored at $-80^{\circ} \mathrm{C}$.

We used a 5' RACE methodology using modified gene-specific primers in the TCRA or TCRB constant region. The purified 5' RACE PCR products were processed to make sequencing libraries using the KAPA Hyper Prep kit. Sequencing was performed using an Illumina MiSeq reagent 500-cycle V2 kit by paired-end $250 \times 2$ cycles. The paired-end reads from the MiSeq were submitted to MiXCR (https://milaboratory.com/software/mixcr/) for TCRA and TCRB rearrangement analyses. The unique CDR3 amino acid sequences (CDR3 clones) for each sample were summarized based on the MiXCR results. Single-copy CDR3 clones were removed. The frequency of a clonotype was calculated by the copy number of the clonotype divided by the total number of copies of all clonotypes in a sample. Bioinformatics processing and further analytics were performed using the Stanford Sherlock supercomputing cluster (https://www.sherlock.stanford.edu/) and customized analysis platform.

Statistics. For statistical comparison of groups in different analysis, we used the 2-tailed Student's $t$ test, with significance assessed at $P<0.05$.

Study approval. This study included 12 patients with high-risk malignancies who had HLAmatched sibling donors (Table 1). The study was approved by the Stanford Institutional Review Board (NCT01660607) and FDA investigational new drug (IND) 14686. Written informed consent was obtained for all patients and donors consistent with the Declaration of Helsinki.

\section{Author contributions}

RSN, GL, and EHM contributed to overall clinical trial design, and GL and EHM served as protocol directors, with SA, LJ, LM, RL, ARR, JS, WKW, DM, CT, and KS contributing to patient care. EHM, GL, RSN, $\mathrm{RA}$, and KT contributed to the IND application submission. Experimental design, analysis, and interpretation were performed by RSN and EHM. Experimental execution and analysis were performed by BX, JB, $\mathrm{KH}, \mathrm{BS}$, SWT, and KM. The manuscript was drafted for important intellectual content by EHM, RSN, and GL. All authors gave their agreement to be accountable for all aspects of the work in ensuring that questions related to the accuracy or integrity of any part of the work are appropriately investigated and resolved.

\section{Acknowledgments}

Support of this study was provided by the NHBLI (R01 HL114591; RSN) and NIH (K08HL119590; EHM). Support from the Doris Duke Foundation in the form of a Distinguished Physician Scientist Award (RSN) that helped develop the approaches used in this study is acknowledged.

Address correspondence to: Everett H. Meyer, Division of Blood and Marrow Transplantation, Stanford University School of Medicine, Stanford, California, USA, 269 West Campus Drive CCSR Building Room 2245C, Stanford, California 94305-5623, USA. Phone: 650.497.1468; Email: evmeyer@stanford.edu.

1. Ramlal R, Hildebrandt GC. Advances in the use of regulatory t-cells for the prevention and therapy of graft-vs.-host disease. Biomedicines. 2017;5(2):23.

2. Edinger M, et al. CD4+CD25+ regulatory T cells preserve graft-versus-tumor activity while inhibiting graft-versus-host disease after bone marrow transplantation. Nat Med. 2003;9(9):1144-1150.

3. Jones SC, Murphy GF, Korngold R. Post-hematopoietic cell transplantation control of graft-versus-host disease by donor CD425 T cells to allow an effective graft-versus-leukemia response. Biol Blood Marrow Transplant. 2003;9(4):243-256

4. Riley JL, June CH, Blazar BR. Human T regulatory cell therapy: take a billion or so and call me in the morning. Immunity. 2009;30(5):656-665.

5. Tang Q, Bluestone JA. Regulatory T-cell therapy in transplantation: moving to the clinic. Cold Spring Harb Perspect Med. 2013;3(11):null.

6. Di Ianni M, et al. Tregs prevent GVHD and promote immune reconstitution in HLA-haploidentical transplantation. Blood. 
2011;117(14):3921-3928.

7. Trzonkowski $P$, et al. First-in-man clinical results of the treatment of patients with graft versus host disease with human ex vivo expanded CD4+CD25+CD127- T regulatory cells. Clin Immunol. 2009;133(1):22-26

8. Trzonkowski $\mathrm{P}$, et al. Treatment of graft-versus-host disease with naturally occurring T regulatory cells. BioDrugs. 2013;27(6):605-614.

9. Nguyen VH, et al. In vivo dynamics of regulatory T-cell trafficking and survival predict effective strategies to control graft-versus-host disease following allogeneic transplantation. Blood. 2007;109(6):2649-2656.

10. Parmar S, et al. Third-party umbilical cord blood-derived regulatory T cells prevent xenogenic graft-versus-host disease. $C y t o t h e r-$ apy. 2014;16(1):90-100.

11. Brunstein CG, et al. Infusion of ex vivo expanded T regulatory cells in adults transplanted with umbilical cord blood: safety profile and detection kinetics. Blood. 2011;117(3):1061-1070.

12. Florek M, et al. Freeze and thaw of CD4+CD25+Foxp3+ regulatory t cells results in loss of CD62L expression and a reduced capacity to protect against graft-versus-host disease. PLoS ONE. 2015;10(12):e0145763.

13. Martelli MF, et al. HLA-haploidentical transplantation with regulatory and conventional T-cell adoptive immunotherapy prevents acute leukemia relapse. Blood. 2014;124(4):638-644.

14. Chaidos A, et al. Graft invariant natural killer T-cell dose predicts risk of acute graft-versus-host disease in allogeneic hematopoietic stem cell transplantation. Blood. 2012;119(21):5030-5036.

15. Schneidawind D, Pierini A, Negrin RS. Regulatory T cells and natural killer T cells for modulation of GVHD following allogeneic hematopoietic cell transplantation. Blood. 2013;122(18):3116-3121.

16. Heinrichs J, Bastian D, Veerapathran A, Anasetti C, Betts B, Yu XZ. Regulatory T-cell therapy for graft-versus-host disease. J Immunol Res Ther. 2016;1(1):1-14.

17. Schlöder J, Berges C, Tuettenberg A, Jonuleit H. Novel concept of CD4-mediated activation of regulatory t cells for the treatment of graft-versus-host disease. Front Immunol. 2017;8:1495.

18. MacDonald KG, et al. Alloantigen-specific regulatory $\mathrm{T}$ cells generated with a chimeric antigen receptor. J Clin Invest. 2016;126(4):1413-1424.

19. Pierini A, et al. T cells expressing chimeric antigen receptor promote immune tolerance. JCI Insight. 2017;2(20):e92865.

20. Mahr B, Granofszky N, Muckenhuber M, Wekerle T. Transplantation tolerance through hematopoietic chimerism: progress and challenges for clinical translation. Front Immunol. 2017;8:1762.

21. Kurtz J, Wekerle T, Sykes M. Tolerance in mixed chimerism — a role for regulatory cells? Trends Immunol. 2004;25(10):518-523. 